\title{
Thoughts on Fiber Optics in Dissolution Testing
}

\author{
Schatz C. ${ }^{1,2}$, Ulmschneider M., ${ }^{1}$ Altermatt R., ${ }^{1}$ Marrer S. ${ }^{1,2}$ and Altorfer H. ${ }^{1,2}$ \\ ${ }^{1}$ Pharmaceutical Quality Control and Quality Assurance, F. Hoffmann-La Roche Ltd., Basel, Switzerland \\ 2 Pharmaceutical Analysis, Swiss Federal Institute of Technology, Zurich, Switzerland
}

email: caspar.schatz@roche.com

\begin{abstract}
Introduction

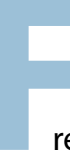
iber optics are a cutting edge and emerging technology for dissolution testing. In situ fiber optic dissolution monitoring has to be regarded as an alternative to conventional dissolution testing where a volume of liquid is extracted from each vessel at appropriate time points. The tedious process of analyzing the sample involves sample filtration before UV/VIS analysis. Since fiber optics allow an in situ measurement without any sample preparation several critical parameters need to be evaluated carefully: the immerision probe design and its positioning in the dissolution vessel, excipient interference correction methods, degree of automation and the spectroscopic assembly. These issues are addressed and discussed in the following sections.
\end{abstract}

\section{Fiber optic immersion probes}

The available immersion probes suitable for dissolution monitoring can be divided into two different design types: prism and mirror probes

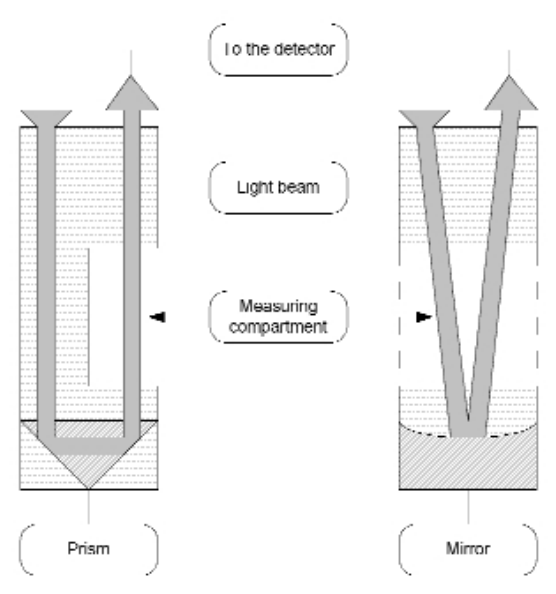

Figure 1 Two different designs of fiber optic immersion probes. In the probe on the left the light beam is guided through the probe by a prism whereas on the right a mirror is used for reflection.

\section{(Figure 1)}

As is shown in Ref.[1], there are substantial differences between the available probes and the probe designs.

Because of their design, which minimize the effects of scattered light, prism probes have a very large linear range. The immersion probe requires a large linear range, depending on the dosage and absorption coefficient of the pharmaceutical, so that it can be used to investigate the dissolution behavior from 0 to a maximum of $120 \%$.

The advantages of the prism type of immersion probe in linearity are, however, associated with an inability to change the optical path length. Because of the precise arrangement of the prism and lenses, it is difficult to conceive a simple method of switching the immersion probe tip for this purpose. However, fixed path lengths are better suited to the determination of $\mathrm{A}(1 \%, 1 \mathrm{~cm})$ values, because changes in path length resulting from removal and replacement of probe tips cannot occur. If the stability of the pharmaceutical permits, quantification can be carried out with the aid of the absorption coefficient, which is less labour intensive than methods of quantification involving standard solutions.

In the case of mirror probes it is possible to change tips, and thus quickly and cheaply change the optical path length to fit the particular requirements. However, probes of this type have a smaller linear range.

\section{Probe positioning in dissolution analysis}

Other factors such as the thickness of the immersion probe and particularly how it is positioned in the vessel must also be taken into account.

In principle, there are four ways of doing this (Table 1).

Table 1. Possible methods of positioning probes in the dissolution vessel

\section{Positioning of the probe}

Whole probe permanently in the vessel

Only in vessel during measurement:

Probe is removed from the medium completely between measurements

Part of the probe permanently in the vessel:

Window in the USP position during measurements, at other times just below the liquid surface

Permanently in a hollow shaft

If the immersion probes remain in the dissolution vessel throughout the dissolution test, particular attention must be paid to the changed hydrodynamic conditions in the dissolution vessel. Thick immersion probes have a larger effect on the hydrodynamics in the vessel than thinner ones [2]. It is, therefore, sometimes a good idea to use special thin immersion probes to minimize the effects on the hydrodynamics. If the influence of immersion probes permanently located in the vessel has any effect at all on the rate of dissolution of the medic- 
inal product concerned, the problems of the resultant changes in the hydrodynamics can also be solved as follows. Immersion probes have a highly reproducible effect on flow behavior [2,3], so this effect can be determined quantitatively. The measured effect can subsequently be taken into account in the form of an offset in the productspecific specification. It is conceivable that $87 \%$ active-ingredient dissolution in 20 min would be required in dissolution tests using optical fibers and immersion probes permanently located in the vessel, whereas only $85 \%$ dissolution would be required over the same period using a conventional method of dissolution testing which does not disturb the hydrodynamics [3].

Another way of minimizing the hydrodynamic effect of immersion probes is to remove the probe from the dissolution vessel after each measurement. By analogy with sample collection in conventional active-substance dissolution tests, the absorption measurement is typically carried out at a point half way between the surface of the test liquid and the top of the paddle stirrer or rotating basket, and at least $10 \mathrm{~mm}$ from the wall of the vessel $[4,5]$. If the immersion probe is only introduced into the vessel for the absorption measurement and is then removed again, the disturbance of the hydrodynamics and the thickness of the immersion probe are not particularly important [2] because, as shown in Ref. [6], there is practically no difference between the use of a conventional cannula to collect a sample and the use of an immersion probe $[6,7]$. Nevertheless, this should be verified for the specific pharmaceutical formulation within the framework of method validation tests.

A compromise between the two positioning methods described above can be achieved if, after each measurement with the probe window half way between the liquid surface and the top of the paddle stirrer or rotating basket, it is lifted until the window is just below the surface of the liquid. This drastically reduces the hydrodynamic effect but, on the other hand, it avoids the formation of air bubbles in the probe window during insertion, which adversely affects the absorption measurement. Drying could result in the collection of residues in the probe window if the immersion probe were removed from the medium completely, especially during longer dissolution tests on extended-release medicinal products.

If removing the immersion probe from the medium between measurements, it should also be remembered that that optical fibers are moved when doing so.This has adverse effects on the precision of the measurement in particular. However, it was also demonstrated that, despite the disadvantage, it is possible to validate methods involving movement of the immersion probe within the framework of normal industrial acceptance criteria $[8,9]$.

If immersion probes are placed in the hollow shaft of the paddle-stirrer and rotating basket apparatus, regulatory requirements mean that the diameter of the shaft containing the probe must not exceed $9.75 \pm$ $0.35 \mathrm{~mm}$ [4]. Although the shaft needs to be modified and fitted with sample windows, the hydrodynamic effects of these changes compared with standard equipment $[4,5]$ are virtually nonexistent $[10,11]$.In addition, the immersion probes are not moved at any time during the dissolution test, which has beneficial effects on the precision of the method.

The pharmaceutical product often breaks down into relatively large particles, especially during dissolution tests on immediate-release tablets. It should be stated that large particles tend to stay at a larger radial distance from the shaft than small particles. The effect of turbidity is thus lowest in the region of the hollow shaft.Clearly, it is possible to compensate for a lower turbidity effect and small particles more reproducibly and more precisely.

\section{Matrix compensation}

As often demonstrated, insoluble constituents of solid pharmaceutical products in the dissolution medium often give rise to a constant offset over a wide wavelength range. An offset which increases or decreases with increasing wavelength is observed less often.This means that relatively simple methods of matrix compensation, e.g., analytical methods with one compensation wavelength, are adequate. Because of the better signal-to-noise ratio, methods which allow for turbidity in the absorption spectrum are to be preferred to derivative spectroscopic methods. This disadvantage of derivative spectroscopy can be partly compensated by the addition of several wavelengths, 


\section{Fiber Optics in Dissolution Testing... continued}

because the noise increases less strongly than the signal when carrying out this sort of addition. When using numerical derivatization algorithms such as Savitzky or Savitzky-Golay, it is possible to choose the number of points to be taken into consideration $[12,13]$.The more points the algorithm takes into account, the greater the smoothing effect on the derivative spectrum. Especially in the case of tablets which break down into large particles during dissolution tests, the smoothing effect may improve the accuracy and reproducibility of the method.

\section{Automation and spectroscopic assembly}

A further aspect of the use of immersion probes in dissolution testing is the degree of automation. When using manual methods, the use of immersion probes can reduce the analysis time [7], because sample collection and the time-consuming sample preparation, involving filtration and transfer of the solution into cells, are not required. Immersion probes also entail lower fixed costs, as filters and disposable syringes are not required.

In semi-automatic and fully-automatic dissolution tests, immersion probes are mainly in competition with systems using automatic filtration and flow-through cells for absorption measurement. Since these systems also operate on-line, the time saving is no longer of major significance. However, the assembly of the apparatus is simpler when using immersion probes, as no pump or filter station are required, which also does away with the need for disposable materials in the form of filters, and the associated suitability test. Typical problems such as sample volume errors and the resultant change in volume in the dissolution vessel, dead volumes, absorption in tubes, filter blockage, and air bubbles in flow-through cells are also eliminated. It is thus easy to see that, viewed overall, immersion probe systems are more robust and require less qualification work than conventional systems involving filtration and flow-through cells.

Depending on the instrumentation used to make measurements in dissolution tests using fiber optics, other important parameters emerge. For example, the first fiber optic dissolution monitoring system which was automated used a single immersion probe which was moved from vessel to vessel by a robot arm $[14,15]$ showed all the advantages of the elimination of the filter station and the pump, but it should be noted that the use of a robot arm involves considerable qualification work. In addition, the data acquisition power when simulta- neously carrying out six tests is relatively low, as the robot arm obviously has to insert the immersion probe sequentially into each of the vessels.

Another approach involves the use of a multiplexer. This instrument can switch the light source and detector between several immersion probes. This increases the data acquisition power, as switching from vessel to vessel is carried out substantially more quickly. However, the multiplexer is a device which requires qualification and which must be shown to be reproducible. It should also be noted that this in fact replaces the pump and filter station. It is thus desirable for the multiplexer to deliver better results in respect of robustness and reproducibility.

Approaches which use a mechanical multiplexer [14] have the advantage that almost $100 \%$ of the intensity of the light source is available in the appropriate channel. On the other hand, switching depends upon the mechanical movement of optical fibers. In addition, the quartz glass fibers need to be positioned very accurately if switching is to be carried out reproducibly (optical fibers typically have a diameter of 200 to $600 \mu \mathrm{m}$ ).

When optical multiplexers are used several immersion probes are coupled with the light source and the detector, shutters ensure that the signal from only one channel reaches the detector at a time. This avoids the mechanical movement and alignment of optical fibers, which has beneficial effects on the robustness of the apparatus. However, the energy of the light source is split, so each channel only receives a fraction of the total intensity. This disadvantage can, however, be counteracted using longer exposure times.

The problems of a multiplexer can also be avoided entirely. For example, a CCD (charge coupled device) chip can be used, the detection surface of the chip being divided into several rows, each row being assigned to a single channel [16, 17]. Alternatively, each vessel can also be equipped with its own detector when carrying out simultaneous dissolution tests on different medicinal products [18]. The resultant advantages in terms of robustness can, however, be reflected in the cost if diode array detectors (DAD) detectors are used. On the other hand, CCD detectors do not have the same spectroscopic quality as diode array detectors [19]. In addition, every detector or channel in such systems has to be qualified separately.

Up to now, the automation of dissolution testing has concentrated on the simultaneous testing of 
several samples (usually six). However, an alternative approach is the complete automation of one vessel, with several test samples being examined sequentially [20]. If optical fibers are used for this purpose, the problems of using a multiplexer can be avoided. In addition, costs only arise for one detector if DAD modules are used for the absorption measurements. There are losses, however, particularly in terms of throughput.

Irrespective of the spectroscopic set-up, the positioning of the immersion probes in the vessel must be taken into consideration if they are to be partly or completely removed from the vessel between absorption measurements. A lifting device will be required in the event of automation, and it will have to be controlled and qualified. Permanent positioning of the immersion probes in the vessel or hollow shaft is substantially easier.

\section{System evaluation}

The following factors have been found to be central in the evaluation of systems for the fiber optic examination of active-substance dissolution: the linear range, the detection range, the robustness, and the software.

As has been shown, the linear range depends partly on the type of immersion probe used and also partly on the type of detector. For example, DAD spectrometers generally have a smaller linear range than scanning spectrometers. In the case of matrix compensation methods, the detectable wavelength range is the most important factor. By contrast, the robustness is decisively influenced by the nature and number of moving parts. Although both a mechanical multiplexer and an optical multiplexer contain moving parts, the mechanical movement and orientation of optical fibers is more critical in terms of robustness than the rotation of a perforated disc. For the user, the software is the most important element of every piece of apparatus, as it permits quality assurance procedures and efficient on-line matrix compensation. The number of matrix compensation methods and other variable parameters the software includes determine the flexibility of the fiber optic dissolution instrument.

In addition, the economic effects of fiber optic systems are also particularly important in the pharmaceutical industry.

Table 1 lists the systems which are currently commercially available (with the exception of the semi-automatic Tidas II).

Please note that the list does not claim to be complete.

Table 2 Fiber optic instruments for the investigation of dissolution behavior

\begin{tabular}{|c|c|c|}
\hline Manual systems & Description & Manufacturer \\
\hline Tidas II & $\begin{array}{l}\text { DAD spectrometer } \\
(190-1020 \mathrm{~nm})\end{array}$ & $\begin{array}{l}\text { J\&M, Aalen } \\
\text { Germany }\end{array}$ \\
\hline Varian Cary 50 & $\begin{array}{l}\text { Scanning spectrometer with fiber- } \\
\text { optic coupler } \\
(190-1100 \mathrm{~nm})\end{array}$ & $\begin{array}{l}\text { Varian, Inc., } \\
\text { Victoria, Australia }\end{array}$ \\
\hline Semi-automatic systems & Description & Manufacturer \\
\hline 10 & $\begin{array}{l}\text { Scanning spectrometer (Varian Cary } 50) \\
\text { with mechanical multiplexer (Cassini) } \\
(190-1100 \mathrm{~nm})\end{array}$ & $\begin{array}{l}\text { C Technologies } \\
\text { Cedar Knolls, USA }\end{array}$ \\
\hline Opt-Diss & $\begin{array}{l}\text { Multi-channel CCD spectrometer } \\
(200-400 \mathrm{~nm})\end{array}$ & $\begin{array}{l}\text { LEAP Technologies } \\
\text { Carrboro, USA }\end{array}$ \\
\hline Rainbow Dynamic Dissolution Monitor ${ }^{\mathrm{TM}}$ & $\begin{array}{l}6 \text { or } 12 \text { DAD detectors } \\
(200-400 \mathrm{~nm})\end{array}$ & $\begin{array}{l}\text { Delphian L.P. } \\
\text { Ardsley, USA }\end{array}$ \\
\hline Tidas II semi-automatic & $\begin{array}{l}\text { In development: } \\
\text { DAD spectrometer (Tidas II) } \\
\text { with optical multiplexer } \\
(190-1020 \mathrm{~nm})\end{array}$ & $\begin{array}{l}\text { J\&M, Aalen } \\
\text { Germany }\end{array}$ \\
\hline ZODIAC & $\begin{array}{l}\text { DAD spectrometer with mechanical } \\
\text { multiplexer } \\
(200-620 \mathrm{~nm})\end{array}$ & $\begin{array}{l}\text { Carl Zeiss Jena GmbH } \\
\text { Jena, Germany }\end{array}$ \\
\hline Fully automatic systems & Description & Manufacturer \\
\hline Zeiss-Zymark robot & $\begin{array}{l}\text { DAD spectrometer (Zeiss MCS 551), } \\
1 \text { immersion probe and robot arm } \\
\text { (Zymark Ltd.) } \\
(200-620 \mathrm{~nm})\end{array}$ & $\begin{array}{l}\text { Carl Zeiss Jena GmbH } \\
\text { Jena, Germany }\end{array}$ \\
\hline
\end{tabular}




\section{Fiber Optics in Dissolution Testing ... continued}

\section{Conclusions}

Since formulations may differ greatly depending on the active ingredient, there is no immersion probe, no analytical matrix compensation technique and no instrument, which is suitable for all problems. The choice of priorities and principal assessment criteria such as matrix compensation methods, immediate-release or extended-release formulations, desired throughput, required linear range, robustness of the apparatus, degree of automation, etc., will produce different ranking orders of the available equipment and techniques.

However, after careful evaluation fiber optics is a beneficial and cost-effective tool for dissolution testing in routine quality control and research environments.

\section{References}

[1] Schatz C., Ulmschneider M., Altermatt R., Marrer S., Altorfer $\mathrm{H}_{\text {., }}$ Testing fibre optic immersion probes for UV/vis spectroscopy, Spectroscopy Europe, 12 (6) 12-18 (2000)

[2] Banakar U.V., Pharmaceutical Dissolution Testing, Marcel Dekker, Inc., New York, Basel, Hong Kong (1992)

[3] Bynum K., New Advances in Fiber Optic Dissolution Testing, Conference Presentation, Dissolution Testing, Tysons Corner (2000)

[4] European Pharmacopoeia, 3rd Edition, Council of Europe, Strasbourg (1996)

[5] USP 24 NF 19, United States Pharmacopeial Convention, Inc., Rockville, MD (1999)

[6] Schatz C., Ulmschneider M., Altermatt R., Marrer S., Hollow Shaft Sampling with Fiber Optics, Dissolution Technologies, 7 (1) 20-21 (2000)

[7] Schatz C., Ulmschneider M., Altermatt R., Marrer S., Altorfer H., Manual In Situ Fiber Optic Dissolution Analysis in Quality Control, Dissolution Technologies, 7 (2) 6-13 (2000)

[8] International Conference on Harmonisation, Validation of Analytical Procedures: Methodology, ICH Harmonised Tripartite Guideline Q2B (1995)

[9] Pharma Switzerland, Quality Assurance and Quality Control. Guideline No.G.BS.115.01, Validation of Analytical Methods, F. Hoffmann-La Roche Ltd., Basel (1998)

[10] Mirza T., Dissolution - A Current USP Perspective, Conference Presentation, Dissolution Testing, Tysons Corner (2000)

[11] Hengst R., Rolli R., Hollow Shaft' Sampling Method in Dissolution Testing, Dissolution Technologies, 6 (1) 16-22 (1999)

[12] Stein E., Algorithm for Smoothing and Derivatives According to Savitzky-Golay, Carl Zeiss Jena GmbH, Jena

[13] Spectralys User Manual for Spectrometers Tidas and Tidas II, J\&M, Aalen (1997)

[14] Fiber Optics in Dissolution Testing with the MCS 500 Diode-array UV-VIS Spectrometer, Spectral Sensors, Carl Zeiss Jena $\mathrm{GmbH}$, Jena

[15] Aldrigde P.K., Melvin D.W., Williams B.A., Bratin K., Kostek L.J., Sekulic S.S., A Robotic Dissolution System with On-
Line Fiber-Optic UV Analysis, Journal of Pharmaceutical Sciences, 84 (8) 909-914 (1995)

[16] Cho J.H., Gemperline P.J. Salt A., Walker D.S., UV/Visible Spectral Dissolution Monitoring by In Situ Fiber-Optic Probes, Analytical Chemistry, 67, 2858-2863 (1995)

[17] Inman G., Martin W., The Use of Fiber Optics Provides a New, Simplified Approach to Dissolution Testing, Pharmaceutical Laboratory, 1 (1) 46 (1998)

[18] Schatz C., Ulmschneider M., Altermatt R., Marrer S., Evaluation of the Rainbow Dynamic Dissolution Monitor $^{\mathrm{TM}}$ Semi-automatic Fiber Optic Dissolution Tester, Dissolution Technologies, 7 (4) 8-17 (2000)

[19] Bynum K, Kassis A., Fiber Optic Dissolution Testing, Delphhian Fiber Optic Seminar, Ardsley (2000)

[20] Introducing the New Automated Dissolution Module (ADM) 399, Source for Automation, Inc., Holliston

\section{Correspondence \\ Caspar Schatz \\ F. Hoffmann-La Roche Ltd. \\ $\mathrm{POBQ}$, Building 61/427 \\ $\mathrm{CH}-4070$ Basel, Switzerland \\ Phone+41-61-687 1433 \\ Fax +41-61-688 8020 \\ caspar.schatz@roche.com}

